\title{
Huygens Principle based UWB Microwave Imaging Method for Skin Cancer Detection
}

\author{
Navid Ghavami \\ School of Engineering \\ London South Bank University \\ London, SE1 0AA, UK \\ Email: ghavamin@1sbu.ac.uk
}

\author{
Gianluigi Tiberi \\ Fondazione IMAGO 7 \\ IRCCS Stella Maris \\ Viale del Tirreno \\ I-56128 Pisa, Italy
}

\author{
Mohammad Ghavami \\ and Sandra Dudley \\ School of Engineering \\ London South Bank University \\ London, SE1 0AA, UK
}

\author{
Majella Lane \\ School of Pharmacy \\ University College London \\ London, WC1N 1AX, UK
}

\begin{abstract}
In recent years, Ultra Wideband (UWB) technology has emerged as a promising alternative for use in a wide range of applications. One of the potential applications of UWB is in healthcare and imaging, motivated by its non-ionizing signals, low cost, low complexity, and its ability to penetrate through mediums. Moreover, the large bandwidth covered by UWB signals permits the very high resolution required in imaging experiments. In this paper, a recently introduced UWB microwave imaging technique based on the Huygens principle (HP), has been applied to multilayered skin model with an inclusion representing a tumor. The methodology of HP permits the capture of contrast such that different material properties within the region of interest can be discriminated in the final image, and its simplicity removes the need to solve inverse problems when forward propagating the waves. Therefore the procedure can identify and localize significant scatterers inside a multilayered volume. Validation of the technique through simulations on multilayered cylindrical model of the skin with inclusion representing the tumor has been performed.
\end{abstract}

\section{INTRODUCTION}

The extremely low power of UWB technology and its large bandwidth makes it a good candidate to solve many problems in various areas including short range high data rate communications and radar applications. In addition to these application schemes, other fields of appropriateness of the UWB technology are under investigation. In particular, interest has grown in the utilization of UWB technology in medicine and healthcare. Recent advances in UWB hardware technology has enabled the possibility of achieving the large bandwidth which is required for higher resolution imaging and detection [1]. In addition, several unique properties of UWB, including its non-ionizing signals, low cost and the ability to penetrate through media (air, skin, bones and tissues) have transformed it into an ideal candidate for a number of healthoriented applications [2].

A number of well established medical imaging techniques are being used in clinics and hospitals today. These methods utilize different technologies and are able to produce welldefined tomographic reconstructions of living tissues using a range of media. Most common are X-ray-based computed tomography (CT), ultrasound scanners, and nuclear magnetic resonance imaging (known as MRI). Beside their advantages, each of these techniques suffer from their own drawbacks, including contrast problems in Ultrasound [3], ionizing radi- ation in CT [4], and the long scan time in the case of MRI [5]. In response to these limitations, microwave imaging has been recognized as a promising non-ionizing and non-invasive alternative screening technology for these application areas.

In general, there are three known types of skin cancer: Squameous Cell Carcinoma (SCC), Basel Cell Carcinoma (BCC), and melanoma. In the majority of cases (especially BCC and SCC), the skin cancers are curable if they are diagnosed at an early stage. For this purpose, the dielectric properties of human tissues can be used as an effective and accurate indicator for diagnostic purposes. [6]. Microwave imaging makes use of the difference in dielectric properties between normal and malignant skin tissues for detection purposes. Human skin comprises of three layers; epidermis, dermis and hyperdermis. With a thickness varying between tissue for 0.1 to $0.5 \mathrm{~mm}$, the epidermis is the outermost layer of the skin. The most external part of the epidermis is a very thin body of dead cells called Stratum Corneum (SC). The thickness of SC varies depending on the location of the skin and it normally ranges between 15 and $150 \mu \mathrm{m}$ [7].

In the past few years, a number of uniform multilayer models have been proposed to simulate the skin layers, with each layer being distinguished by a set boundary and specific electrical properties (permittivity and conductivity) [6], determined by those of free water content [8]. There is an increase in the amount of water content in all types of skin cancer, most notably in the epidermis layer. For malignant tumors and lesions, the water content of the epidermis can reach up to $81 \%$ [9]. These changes in the water content result in a change in the permittivity value of the tissue.

Currently, the most common and accurate approach for skin cancer diagnosis is via the biopsy technique, in which the skin lesion is removed and examined in a laboratory [10]. The diagnostic results usually take around a week to become available and are not guaranteed to be accurate. Meanwhile, reflectometer techniques [10] use millimeter wave guides to discriminate malignant and normal lesions. On the other hand, the approach presented in this paper is a complete imaging technique which allows us to image the tissues and to localize the inclusions. To the best of our knowledge this method has not yet been applied for the application of skin cancer detection. 
For this purpose, the aim of this paper is to apply a novel, fast, and accurate UWB microwave imaging method based on the Huygens Principle (HP) [11] for treating multilayered model with inclusions. Using HP removes the need to solve inverse problems and, consequently, no matrix generation/inversion is required.

Furthermore, UWB allows the utilization of all the information in the frequency domain by combining the information from the individual frequencies, resulting in the construction of a consistent image. It follows that the methodology can identify the presence and location of significant scatterers inside a multilayered volume without having apriori knowledge of the dielectric properties of the target object. Validation of the technique through simulations has been performed, and results are presented, illustrating the effectiveness of the proposed method. Potential applications of the method are skin cancer detection, internal organ and whole body imaging.

In the next section of this paper, the HP-based procedure is reviewed and summarized. The method is the validated through realistic skin simulations in Section III, while Section IV concludes the paper.

\section{The Huygens Principle-Based Procedure}

The procedure employed in this section was first introduced and presented in [11], and was subsequently tested successfully through a number of simulations and measurements for the purpose of breast cancer detection, published in the following papers [12], [13], [14]. To assist reader understanding, some fundamental works presented in [11] is reused here.

As a simple example, consider a cylinder in free space illuminated by a transmitting line source $\mathrm{tx}_{m}$, and operating at a frequency $f$. At this point, it is assumed that the dielectric properties of the bulk of the cylinder are known. The problem consists of identifying the presence and location of discontinuities by using only the fields measured at the receivers outside the cylinder (fig. 1).

Assume that the field at the surface points $r x_{n p}=\left(a_{0}, \phi_{n p}\right)$ is known, such that:

$$
\left.E\right|_{r x_{n p}}=E_{n p} \quad \text { with } \quad n p=1, \ldots N_{P T}
$$

where $N_{P T}$ indicates the number of measured points across the outside cylinder. Recalling the HP, we note that: each locus of a wave excites the local matter which reradiates a secondary wavelets, and all wavelets superpose to a new, resulting wave (the envelope of those wavelets), and so on [15]. Making use of this theory, we calculate the field inside the cylinder as the superposition of the fields re-radiated by the $N_{P T}$ receiving antennas of (1):

$$
E_{\mathrm{HP}}^{\mathrm{rcstr}}(\rho, \phi ; t x ; f)=\sum_{n p=1}^{N_{P T}} E_{n p} G\left(k_{1}\left|\vec{\rho}_{n p}-\vec{\rho}\right|\right)
$$

$(\rho, \phi) \equiv \vec{\rho}$ is the observation point and $k_{1}$ represents the wave number for the media constituting the cylinder. In (2), the string "rcstr" indicates the reconstructed internal field, while

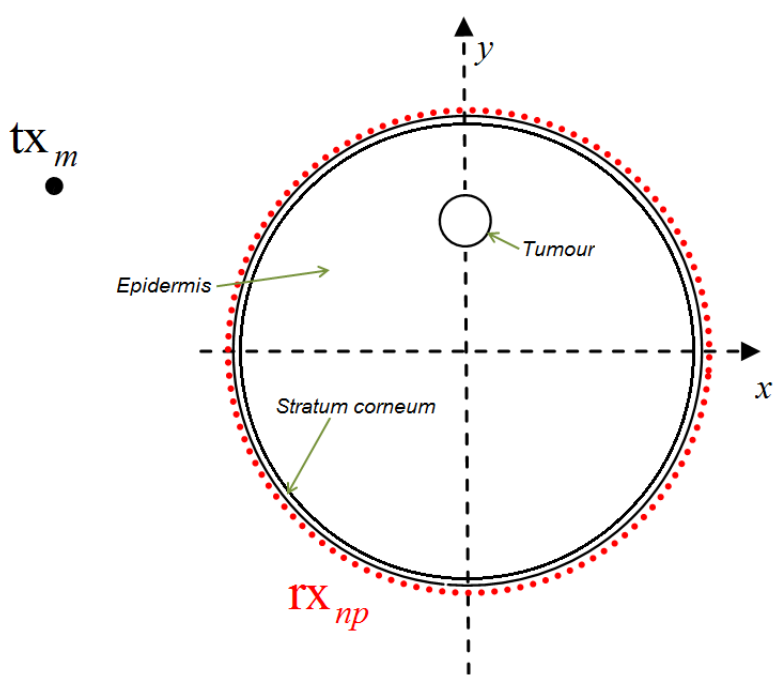

Fig. 1: Pictorial view of the skin cancer detection problem.

the subscript HP refers to the Huygens principle method, and $\operatorname{avg}_{M}\left\{E_{n p}\right\}$ represents the average of signals obtained illuminating the object using $M$ different transmitter positions.

Moreover, $G\left(k_{1}\left|\vec{\rho}_{n p}-\vec{\rho}\right|\right)$ is the Green's function defined as [11]:

$$
G\left(k_{1}\left|\vec{\rho}_{n p}-\vec{\rho}\right|\right)=\frac{1}{4 \pi\left|\vec{\rho}_{n p}-\vec{\rho}\right|} e^{-j k_{1}\left|\vec{\rho}_{n p}-\vec{\rho}\right|}
$$

During the initial simulations, it was observed that an image of the transmitter appeared in the result, which sometimes masked the area of interest. However, this transmitter image can be successfully removed by modifying (2) such that:

$$
E_{\mathrm{HP}}^{\mathrm{rcstr}}(\rho, \phi ; t x ; f)=\sum_{n p=1}^{N_{P T}}\left(E_{n p}-\operatorname{avg}_{M}\left\{E_{n p}\right\}\right) G\left(k_{1}\left|\vec{\rho}_{n p}-\vec{\rho}\right|\right)
$$

where $\operatorname{avg}_{M}\left\{E_{n p}\right\}$ represents the average of signals obtained illuminating the object using $M$ different transmitter positions. This in effect "smears" out the transmitter image.

Thus, assuming $M$ transmitting sources $\mathrm{tx}_{m}, m=1,2 \ldots M$, and $N_{F}$ frequencies $f_{i}$, the intensity of the resulting image $I$ using the Green's functions can be obtained through the incoherent summation:

$$
I_{\mathrm{HP}}(\rho, \phi)=\frac{1}{B} \sum_{m=1}^{M} \sum_{i=1}^{N_{F}} \Delta_{f}\left|E_{H P}^{r c s t r}\left(\rho, \phi ; t x ; f_{i}\right)\right|^{2}
$$

In (5), $\Delta_{f}$ and $B$ represent the frequency and the bandwidth, respectively. The resolution is expected to reach the optical resolution limit of $\lambda_{f_{\max }} / 4$, where $\lambda_{f_{\max }}$ represents the wavelength in the cylinder calculated at the highest frequency; this gives the rule of thumb for determining the highest frequency to be used. 


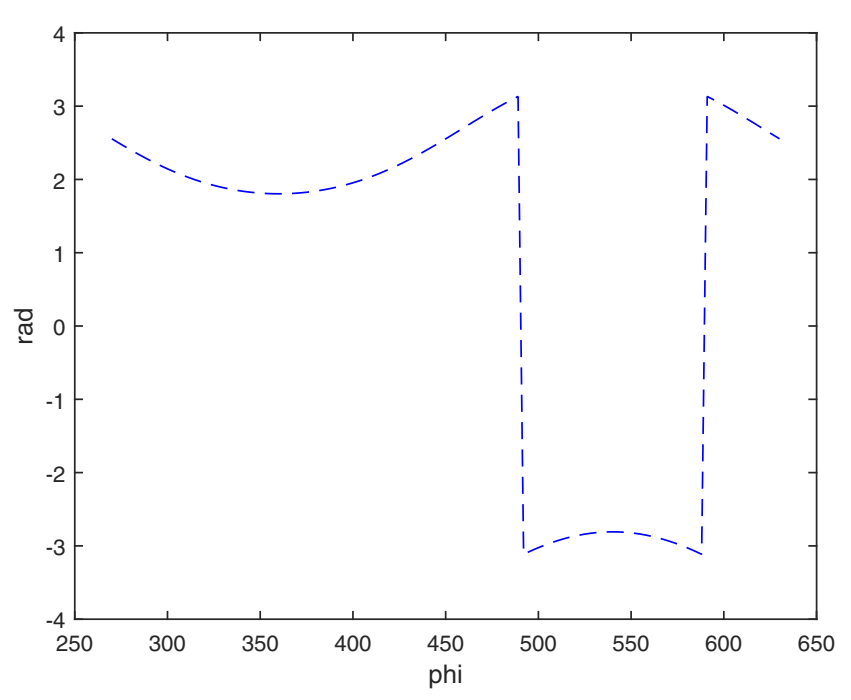

Fig. 2: Phase of the field on the external surface.

\section{VALIDATION THROUGH SIMULATIONS:}

\section{MULTILAYERED MODEL WITH AN ECCENTRIC INCLUSION}

The applicability of the analytical formulation presented in [13] is tested by simulating a 3-layer cylindrical model with an eccentric inclusion. The model, which is $2.5 \mathrm{~mm}$ in radius has realistic human skin tissue properties assigned to its three layers. The external cylinder is assumed to be a $0.1 \mathrm{~mm}$ thick Stratum Corneum, with $\varepsilon_{r 1}=10$ and $\sigma_{1}=0.0001 \mathrm{~S} / \mathrm{m}$ [16]. The internal concentric cylinder and the eccentric inclusion are then assigned the electrical properties of Epidermis $\left(\varepsilon_{r 1}=33\right.$ and $\left.\sigma_{1}=1 \mathrm{~S} / \mathrm{m}\right)$ [17] and malignant tumor $\left(\varepsilon_{r 1}=50\right.$ and $\sigma_{1}=$ $3 \mathrm{~S} / \mathrm{m}$ ) [18] respectively. Furthermore, the eccentric inclusion is $0.5 \mathrm{~mm}$ in radius and its center is placed at $2 \mathrm{~mm}$ deep from the external layer of the skin, $90^{\circ}$ degrees relative to the $\mathrm{X}$-axis.

Four transmitter sources placed $1 \mathrm{~cm}$ from the axis of the cylinder illuminate the external cylinder, while a frequency band of $1-10 \mathrm{GHz}$ with frequency spacing of $10 \mathrm{MHz}$ is used in the computer simulation. For each illuminating source and for each frequency, the field $\left(E_{n p}\right)$ at $N_{P T}=120$ points lying on the external surface is calculated. Finally, the average of the 4 data sets $\left(\operatorname{avg}_{M}\left\{E_{n p}\right\}\right)$ is calculated and HP is applied to the difference between the measured field and the average field as stated in (4).

Figures 2 and 3 show the variation in phase and magnitude of the measured field on the external surface of the cylinder, respectively, while fig 4 depicts the normalized intensity adjustment obtained through (5) and using an appropriate image adjusting. The image is adjusted by forcing the intensity values below 0.7 up to zero and expanding from 0 to 1 values above 0.7 . A bright peak representing the tumor can be clearly observed and detected in the region of the inclusion with a resolution of $0.6 \mathrm{~mm}$.

It can be observed that the method successfully detects and locates the skin tumor in this simulation. A malignant tumor has electrical properties higher than most human tissues [19],

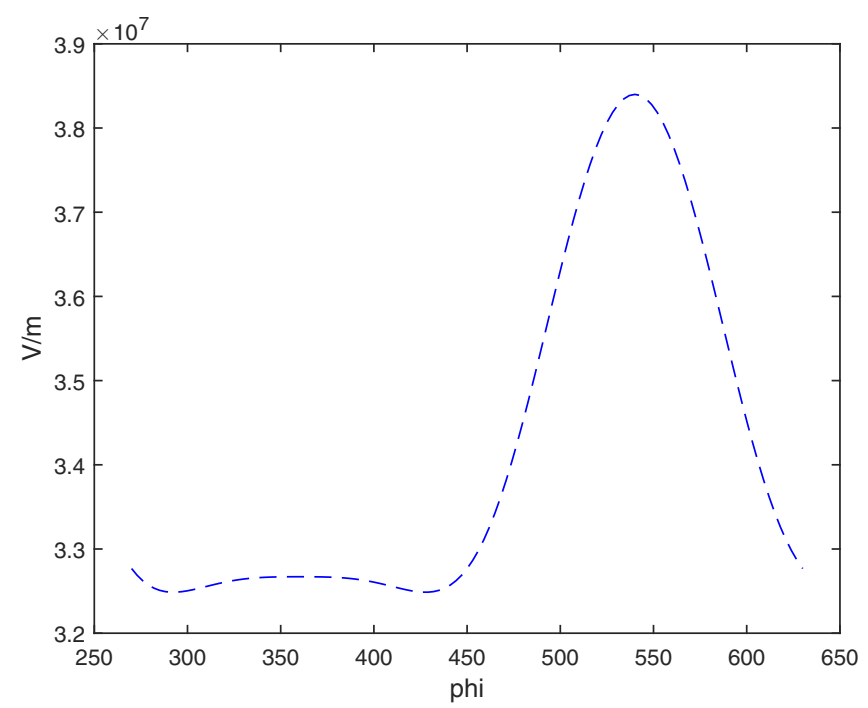

Fig. 3: Magnitude of the field on the external surface.

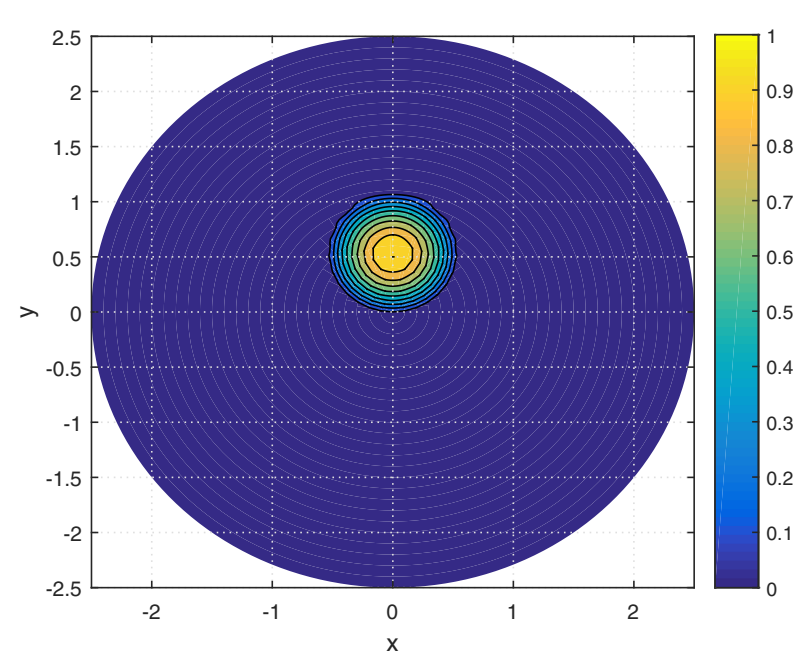

Fig. 4: Cylinder with an inclusion: normalized intensity obtained through (5). All scales are in millimeters.

hence this result would suggest its detection would be achieved in almost all cases in various parts of the body.

The effect of the change in the permittivity of the epidermis and that of the malignant tissue was then investigated. This was done to consider the change in the permittivity of the epidermis and the tumor caused by the amount of water content [18], and to take into account the alternative permittivity values of these tissues presented by other researchers [16]. More in detail, we were interested to see if these changes in permittivity values would affect the resolution and localization of the detected tumor. The results are tabulated in Table 1 .

It can be seen that the change in the permittivity value of the malignant tumor does not have any affect on the resolution and positioning of the imaging results, while a similar change in the permittivity value of the epidermis results in a very negligible offset and resolution. 


\begin{tabular}{|l|c|c|}
\cline { 2 - 3 } \multicolumn{1}{c|}{} & $\begin{array}{c}\text { Resolution } \\
(\mathrm{mm})\end{array}$ & $\begin{array}{c}\text { Offset } \\
(\mathrm{mm})\end{array}$ \\
\hline$\varepsilon_{\text {epidermis }}=33, \varepsilon_{\text {malig }}=40$ & 0.6 & 0 \\
\hline$\varepsilon_{\text {epidermis }}=33, \varepsilon_{\text {malig }}=60$ & 0.6 & 0 \\
\hline$\varepsilon_{\text {epidermis }}=20, \varepsilon_{\text {malig }}=50$ & 0.7 & 0.1 \\
\hline$\varepsilon_{\text {epidermis }}=40, \varepsilon_{\text {malig }}=50$ & 0.65 & 0.05 \\
\hline
\end{tabular}

TABLE I: Comparison of the imaging results obtained through various permittivity value combinations.

\section{CONCLUSiON}

The application of a recently developed UWB microwave imaging algorithm based on the Huygens principle on realistic skin simulations has been analyzed. The multilayered simulated model assumes realistic skin dimensions and electrical properties for individual layers and the tumor. Forward-propagation of the waves using the HP procedure removes the need to solve inverse problems and matrix generation/inversion. This simple methodology benefits from the capability of capturing the contrast (dielectric variation). The method allows all the information in the frequency domain to be utilized by combining the information from the individual frequencies to construct a consistent image. HP method is able to provide a resolution of approximately one quarter of the shortest wavelength in the dielectric medium, while having bandwidths and power levels satisfying UWB rule and power safety limits, respectively. It has been shown that the HP can detect and locate an inclusion in a multilayered cylindrical skin model. Measurements on realistic skin models for further validation of the methodology are currently under investigation.

\section{REFERENCES}

[1] M. Hämäläinen, A. Taparugssanagorn, R. Tesi, and J. Iinatti, "Wireless medical communications using UWB," in ICUWB 2009: IEEE International Conference on Ultra-Wideband, September 2009.

[2] E. M. Staderini, "UWB radar in medicine," IEEE Aerospace and Electronic Systems Magazine, vol. 17, no. 1, pp. 13-18, January 2002.

[3] C. R. Hill and G. R. Ter Harr, "Review article: High intensity focused ultrasound-potential for cancer treatment," The British Journal of Radiology, vol. 68, no. 816, pp. 1296-1303, December 1995.

[4] Principle of Computerized Tomography, R. F. Cotellessa, Ed. et al., IEEE Free Press, 1987.

[5] Mammography and Beyond: Developing Techniques for the Early Detection of Breast Cancer. Washington, DC: Institute of Medicine, National Academy Press, 2000.

[6] S. I. Alekseev and M. C. Ziskin, "Human skin permittivity determined by millimeterwave reflection measurements", Bioelectromagnetics, 28, pp 331-339, July 2007.

[7] M. Egawa, T. Hirao and M. Takahashi, "In vivo estimation of stratum corneum thickness from water concentration profiles obtained with Raman spectroscopy", Acta Dermato-Venereologica, 87, pp. 4-8, 2007.
[8] Y. Feldman, A. Puzenko1, P. B. Ishai1, et al., "The electromagnetic response of human skin in the millimetre and submillimetre wave range", Physics in Medicine and Biology, 54, pp. 3341-3363, 2009.

[9] K. Chand, P. Mehta, D. G. Beetner, R. Zoughi and W. V. Stoecker, "Microwave reflectometry as a novel diagnostic method for detection of skin cancers". Instrumentation and Measurement, pp. 1425-1428, Ottawa, 2005.

[10] A. Taeb, S. Gigoyan and S. Safavi-Naeini, "Millimetre-wave waveguide reflectometers for early detection of skin cancer," Microwaves, Antennas \& Propagation, IET, vol.7, no.14, pp.1182-1186, November 19, 2013.

[11] N. Ghavami, G. Tiberi, D. J. Edwards, and A. Monorchio, "UWB Microwave Imaging of Objects With Canonical Shape," IEEE Transactions on Antennas and Propagation, vol.60, no.1, pp. 231-239, January 2012.

[12] G. Tiberi, N. Ghavami, D. J. Edwards and A. Monorchio, "Ultrawideband microwave imaging of cylindrical objects with inclusions," Microwaves, Antennas \& Propagation, IET , vol.5, no.12, pp.1440-1446, September 2011.

[13] N. Ghavami, G. Tiberi, D. J. Edwards, A. Safaai-Jazi, and A. Monorchio, "Huygens principle based imaging of multilayered objects with inclusions," Progress In Electromagnetics Research B, vol. 58, pp. 139-149, 2014.

[14] N. Ghavami, P. Probert Smith, G. Tiberi, D. J. Edwards and I. Craddock, "Non-iterative beamforming based on Huygens principle for multistatic ultrawide band radar: application to breast imaging," in Microwaves, Antennas \& Propagation, IET , vol. 9, no. 12, pp. 1233-1240, September 2015.

[15] P. Enders, "Huygens principle as universal model of propagation," LatinAmerican Journal of Physics Education, vol. 3, no. 1, pp. 1932, January 2009.

[16] A. Megej, A. Caduff and M. Talary, "Method and device for characterizing the effect of a skin treatment agent on skin," US Patent US20110144525 A1, June 2011.

[17] K. Sasaki and K. Wake and S. Watanabe, "Measurement of the dielectric properties of the epidermis and dermis at frequencies from $0.5 \mathrm{GHz}$ to 110 GHz", Physics in Medicine and Biology, vol. 59, no. 16, pp. 47394747, August 2014.

[18] R. Aminzadeh, M. Saviz and A. A. Shishegar, "Dielectric properties estimation of normal and malignant skin tissues at millimeter-wave frequencies using effective medium theory," 22nd Iranian Conference on Electrical Engineering (ICEE), pp.1657-1661, May 2014.

[19] C. Gabriel, S. Gabriely and E. Corthout, "The Dielectric Properties of Various Tissues" Physics in Medicine and Biology, vol. 41, p.p. 22312249, 1996. 